\title{
Conservative Management of Single-twin Death at 22 Weeks' Gestation at A Tertiary Hospital in North-central Nigeria: A Case Report and Review of Literature
}

\author{
Eka Po ${ }^{1,}$, , Swende Tz ${ }^{1}$, Ojabo Oa ${ }^{1}$, Hembah-Hilekaan $\mathrm{Sk}^{1}$, Ornguze $\mathrm{Aa}^{1}$, Dabit $\mathrm{Oj}^{2}$, \\ Maanongun $\mathrm{Mt}^{1}$, Okoh $\mathrm{Ea}^{1}$, Adia $\mathrm{Jt}^{1}$ \\ ${ }^{1}$ Department of Obstetrics and Gynaecology, Benue State University Teaching Hospital, Makurdi, Nigeria \\ ${ }^{2}$ Department of Paediatrics, Benue State University Teaching Hospital, Makurdi, Nigeria
}

Email address:

ekapeteronche@gmail.com (E. Po)

${ }^{*}$ Corresponding author

\section{To cite this article:}

Eka Po, Swende Tz, Ojabo Oa, Hembah-Hilekaan Sk, Ornguze Aa, Dabit Oj, Maanongun Mt, Okoh Ea, Adia Jt. Conservative Management of Single-twin Death at 22 Weeks' Gestation at A Tertiary Hospital in North-central Nigeria: A Case Report and Review of Literature. Journal of Gynecology and Obstetrics. Vol. 8, No. 4, 2020, pp. 113-116. doi: 10.11648/j.jgo.20200804.18

Received: July 18, 2020; Accepted: July 29, 2020; Published: August 20, 2020

\begin{abstract}
Background: Single-twin intrauterine death in the second and third trimesters poses a great concern and psychological stress to both the parents and the obstetrician. A multidisciplinary approach to conservative management is associated with improved perinatal outcome for the surviving twin. Case: We present a 30-year-old gravida 2 para 1 ( 1 alive). She had an emergency caesarean section during her first delivery. Having been referred from a military hospital, she presented at 22 weeks with single-twin intrauterine death. She was admitted and discharged after one week. Subsequently, she was managed conservatively and had weekly antenatal follow-up visits. She kept a daily fetal kick chart, had fortnightly ultrasound scans for fetal growth and wellbeing and weekly maternal clotting profile. At 37 weeks of gestation, she had a successful repeat caesarean delivery of a live, male neonate with a birth weight of 3.9kg and Apgar scores of 9 at one minute and 10 at five minutes. The remains of the dead co-twin (fetus papyraceus) were seen attached to the placenta. Follow-up by the neonatologist showed that his developmental milestones were normal and comparable to those of his singleton peers. Conclusion: The management of single-twin intrauterine death after the second trimester is psychologically tasking, requiring adequate counselling for the couple. The multidisciplinary approach, adopted in this study, improved perinatal outcome for the surviving co-twin after 15 weeks of conservative management. Prolonged paediatric follow-up of the survivor was imperative.
\end{abstract}

Keywords: Single-twin Intrauterine Death, Conservative Management, Multidisciplinary Approach, Chorionicity, Maternal Coagulopathy, Fetus Papyraceus

\section{Introduction and Literature Review}

Pregnancies with twins are high risk when compared with singleton pregnancies in terms of increased perinatal mortality, and have a high risk of single intrauterine death (SIUD), complicating up to $6 \%$ of twin pregnancies after the second trimester [1-4]. The incidence of single twin demise has also been reported to be $5 \%$ in of all twin pregnancies. The intrauterine fetal death of one twin in the first trimester is common and is known as a "vanishing twin". Around $23 \%$ of twin pregnancies diagnosed at six weeks' gestation will be singleton by 12 weeks. Although a "vanishing twin" may present with vaginal bleeding or mild cramping, it is not associated with adverse perinatal outcome for the surviving twin $[5,6]$. Single-twin demise in the second and third trimesters is less common and varies widely in reported series from $0.5 \%-11.6 \%$ [7]. However, fetal demise occurring after mid gestation (17 weeks gestation) may increase the risk of intrauterine growth restriction (IUGR), preterm labour, preeclampsia, and perinatal mortality in the surviving twin [8]. It may also increase the risk of neurological complication in the surviving twin and is a cause of great concern and psychological stress to both parents and the obstetrician 
[9].

The cause of single twin demise in the majority of cases is unknown. However, twin-to-twin transfusion syndrome, Rh incompatibility, chromosomal and congenital abnormalities, cord round the neck, cord accidents, uterine malformations, velamentous insertion of the cord, placental insufficiency and maternal diseases such as preeclampsia and diabetes mellitus may be implicated $[6,10]$.

Chorionicity is the major determinant of the risk of morbidity and mortality of the surviving co-twin in single twin demise. A systematic review found that following the death of one twin after the first trimester, the odds of intrauterine death of the co-twin and neurological abnormality among survivors were six and four times higher in monochorionic (MC) compared with dichorionic (DC) pregnancies [11]. The "haemodynamic imbalance theory" states that vascular anastomoses (frequently present in monochorionic placentas) allow shunting of blood from the surviving twin to the dead co-twin giving rise to periods of hypoperfusion, hypotension, anaemia and multiorganischaemia and neurological damage in the surviving twin. In dichorionic pregnancy, these vascular anastomoses are not present, but the intrauterine environment that may have caused the initial fetal demise (infection, maternal medical disease) may place the surviving twin at risk as well. Hence, determining the type of placentation by ultrasonography can help in predicting the outcome $[8,9,12$, 13]. Unlike the case of intrauterine fetal death in singletons, maternal disseminated intravascular coagulation (DIC) is extremely rare or never existent in multiples, for some unclear reason $[8,9,14,15]$.

Cattanach [16] et al, Kilby [5] et al and Prompeler [17] et al maintained that fetal outcome is mainly gestational age dependent and made a case for conservative management as opposed to aggressive management, with a view to prolonging pregnancy.

Once conservative manage is chosen, close surveillance of the survivor should be performed using non-stress test, sonographic biophysical profile and assessment of fetal growth [18]. These should be performed along with fetal kick chart and regular maternal coagulation profile.

\section{Case Report}

Mrs. O. K was a 30-year-old gravida 2 para 1 (1 alive) who was at 22 weeks' gestation with twin pregnancy. She was referred from a military hospital with reduced fetal movements, noticed two days prior to presentation to our facility. She had caesarean delivery in her first confinement owing to malposition (persistent occipitoposterior position). She had an ultrasound scan at 8 weeks gestation which showed 2 live fetuses with dichorionic diamniotic placentation.

She was anxious, not dehydrated, anicteric, afebrile $\left(36^{\circ} \mathrm{C}\right)$ and was not pale. Her pulse rate was 84 beats per minute and the blood pressure was $100 / 60 \mathrm{mmHg}$.

The fundal height was 26 centimetres. She had an obstetric ultrasound scan that showed a live fetus at 22 weeks 3 days and a dead co-twin at 21 weeks 2 days with collapsed skull bones and absent heart activity. The estimated gestational age at demise of the co-twin was based on its femur length measurement.

Her packed cell volume was $33 \%$; total white blood cell count was $6 \times 10^{9} / \mathrm{L}$; platelet count was $285 \times 10^{9} / \mathrm{L}$; blood group was $\mathrm{A}$ Rh-positive; haemoglobin genotype was AA. Her HIV I and II, Hepatitis B surface Antigen, Anti-Hepatitis $\mathrm{C}$ Virus andVenereal Disease Research Laboratory tests were normal. Other investigations performed were malarial parasites; urine microscopy, culture and sensitivity; serum electrolytes, urea and creatinine and glucose tolerance test. All of the results were normal.

Her base-line clotting profile was normal with an international normalized ratio (INR) of 0.86 ( $\mathrm{PT}=12 / 14$; APTT=38/44).

The couple was counselled on the risks, benefits and complications which might be associated with the options of management. They opted for conservative as opposed to aggressive management. The neonatologists, haematologists, anaesthetists and theatre were informed about the case and asked to co-manage with us. She was admitted, stabilized and discharge after one (1) week. She was booked for antenatal care and had subsequent weekly follow-up visits until delivery. She was free to get in touch with us whenever it was necessary, throughout the period of conservative management.

She had close maternofetal surveillance. She was taught to keep a daily fetal kick chart which remained normal throughout the period of surveillance. Fortnightly ultrasound was done for fetal growth assessment. From 32 weeks' gestation a weekly biophysical profile (BPP) and non-stress test (NST), which yielded a score of $10 / 10$ on each occasion, were performed. Subsequent weekly clotting profile performed on the mother was normal. At 32 weeks' gestation, she had parenteral dexamethasone given to accelerate fetal lung maturity. Her weekly packed cell volume estimation was normal. At 36 weeks' gestation, ultrasonography documented a live singleton fetus in cephalic presentation and in longitudinal lie, with an estimated weight of $3.56 \mathrm{~kg}$. The placenta was fundal and the liquor volume was adequate for the gestational age. Two units of blood were grouped and saved throughout the period of conservative management.

At 37 weeks' gestation she had a repeat caesarean delivery (on her request). She was delivered of a live male neonate with a birth weight of $3.9 \mathrm{~kg}$ and Apgar scores of 9 at one minute and 10 at 5 minutes. The placenta was sent for histopathological evaluation (it was dichorionic with an identifiable separating membrane). The dead co-twin (fetus papyraceus) was identified along with its umbilical cord connection to the fused dichorionic placentas (figures 1 and 2).

The neonate was declared grossly normal by the neonatologist and has been followed-up by the paediatric department of our facility for the past one and half years. His developmental milestones have been satisfactory in comparison to those of his singleton peers. There was no 
obvious neurological abnormality.

Informed written consent was obtained from the couple to publish this article.

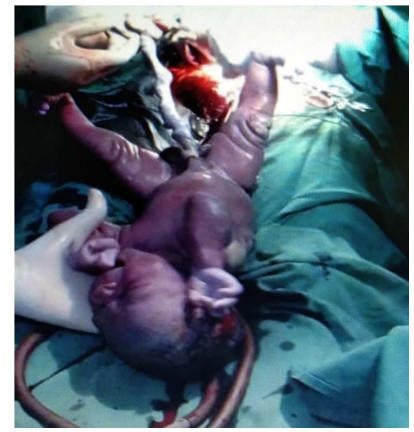

Figure 1. The surviving co-twin delivered by caesarean section.
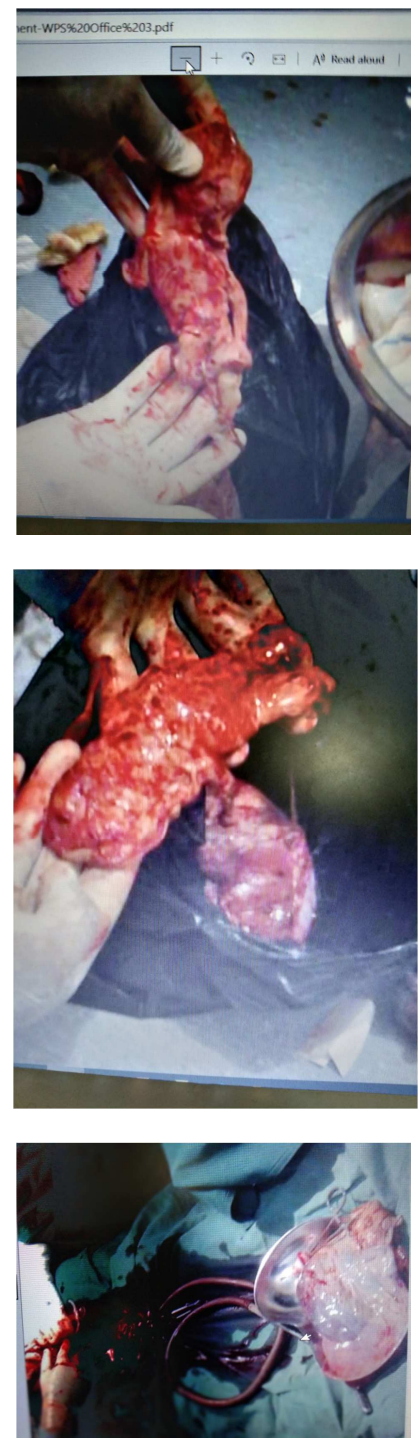

Figure 2. The placenta and the dead co-twin (fetus papyraceus).

\section{Discussion}

This was a case of single-twin demise at 22 weeks, which was managed conservatively with successful delivery of the surviving co-twin at 37 weeks' gestation. This was less than the gestational ages at single-twin demise of 25 weeks and 32 weeks reported by Swarankar [9] et al and Babah [15] et al respectively. The duration of 15 weeks (105 days) of conservation management was one of the longest reported comparable to intervals of 118 days and 108 reported by Baweja [19] et al.

A multidisciplinary approach and counselling was adopted in the conservative management of this case. Parental anxiety may be an important factor in persuading the obstetrician to intervene [10]. The mounting psychological stress that occurred in the couple while anxiously awaiting the delivery of the surviving twin was addressed with regular counselling.

It was a dichorionic twin pregnancy complicated by single intrauterine twin demise. This could be responsible for the favourable prognosis and fetal outcome associated with conservative management of this case.

Two meta-analysis reported that the risk of monochorionic (MC) and dichorionic (DC) co-twin death was $12 \%$ and $4 \%$ respectively; the odds of the surviving co-twin's death following single intrauterine fetal death after 20 weeks of gestation was six times higher in MC than in DC twins; the risk of neurological abnormality in the surviving $\mathrm{MC}$ and $\mathrm{DC}$ co-twins was $18 \%$ and $1 \%$ respectively $[11,20]$. Bajoria [21] et al attributed the increased mortality and morbidity in MC twins after single-twin demise to the presence of placental superficial arterioarterial or veno-venous anastomoses.

She did not develop coagulopathy throughout the duration of conservative management which was in agreement with findings from other case report [8, 9, 14, 15]. However, Landy [22] et al reported a maternal coagulopathy incidence of $25 \%$. Romero [14] et al cited maternal coagulopathy leading to gingival haemorrhage at 29 weeks' gestation.

Mrs. O. K had a repeat caesarean section at 37 completed weeks. We opted for this route of delivery because she had insisted on repeat caesarean to save her further anxiety and psychological stress, which may be associated with trial of labour after caesarean section. Single intrauterine death per se in twin pregnancy is not an indication for caesarean, except in monoamniotic twins which have a $25 \%$ risk of cord entanglement or knotting [16].

\section{Conclusion}

The management of single-twin demise in the second and third terms requires adequate counselling and psychological support for the couple. The multi-disciplinary approach adopted in this study improved outcome and led to the delivery of a healthy, surviving co-twin after 15 weeks of conservative management. Apart from chorionicity, gestational age is a key determinant of perinatal outcome. Conservative management prolonged the pregnancy and increased the gestational age at delivery (in this case from 22 weeks to 37 weeks) and reduced the risk of prematurity. Prolonged, postnatal follow-up of the surviving twin by the neonatologist was imperative. 


\section{Financial Support}

The authors did not receive sponsorship from any source.

\section{Conflict of Interest}

The authors declare that they have no competing interests.

\section{References}

[1] Kleinman JC, Fowler MG, Kessel SS. Comparison of infant mortality among twins and singletons: United States 1960 and 1983. Am J. Epidemiol. 1991; 133 (2): 133-143.

[2] National Institute for Health and Clinical Excellence. Multiple pregnancy: management of twin and triplet pregnancies in the antenatal period. NICE clinical guidance 129; September, 2011. London, England. National Institute for Health and Clinical Excellence, 2011.

[3] Ong S, Zamora J, Khan K, et al. Single Twin Demise: Consequences for the Survivor. In Baker P, Critchley H (eds). Multiply Pregnancy. RCOG Press, 2006; 149-165.

[4] Conte G, Righini A, Griffith PD, Rustico M, Lanna M, Mackie FL, et al. Brain-injured survivors of monochorionic twin pregnancies complicated by single intrauterine death: MR findings in a multicentre study. Radiology. 2018; 288 (2): 582-590.

[5] Kilby MD, Govind A, O'Brien PM. Outcome of twin pregnancies complicated by a single intrauterine death: a comparison with viable twin pregnancies. Obstet Gynecol. 1994; 84: 107-109.

[6] Tunc SY, Agacayak E, Goruk NY, Icen M, Findik FM, Evsen MS, et al. Single intrauterine demise in twin pregnancies; Analysis of 29 cases. Turk J Obstet Gynaecol. 2015; 12 (4): 226-229.

[7] Mark P. Umstad. Obstetric Management Update: Fetal death of a twin. Ages XXIX Annual Scientific Meeting 2019: Perfection, Professionalism and problems. O \& G Magazine. $7^{\text {th }}-9^{\text {th }} \quad$ March, 2019, Crown Towers, Perth (http://www.ogmagazine.org.au/linkout/8373).

[8] Jain D, Purohit RC. Review of twin pregnancies with single fetal death: Management, maternal and fetal outcome. J Obstet Gynaecol India. 2014; 64 (3): 180-183.

[9] Swarankar ML, Shekhawat V, Choudhary M, Choudhary V. Maternal and neonatal outcome of surviving twin after single fetal demise at 25 weeks. A rare case Report. Fertil Sci Res. 2017; 4: 30-34.
[10] Woo HH, Sin SY, Tang LC. Single foetal death in twin pregnancies: review of the maternal and neonatal outcomes and management. Hong Kong Med J. 2000; 6 (3): 293-300.

[11] Ong SSC, Zamora J, Khan KS, Kilby MD. Prognosis for the co-twin following single-twin death: a systematic review. BJOG 2006; 113: 992-998.

[12] Bajoria R, Kingdom J. The case for routine determination of chorionicity and zygosity in Multiple Pregnancy. Prenat Diag. 1997; 17: 1207-1225.

[13] Masheer S, Islam Z, Dileep D, Munim S. (2017). Twin chorionicity and prospective stillbirth risk: experience at a tertiary care hospital. Journal of the Pakistan Medical Association; 67 (3): 360-364.

[14] Romero R, Duffy TP, Berkowitz RL, Chang E, HobbinsJC. Prolongation of a Preterm Pregnancy complicated by death of a single twin in utero and disseminated intravascular coagulation. Effects of treatment with heparin. N Eng J Med. 1984; 310: 772-774.

[15] Babah OA, Olamijulo A, Ayanbode OS, Sanusi MM. Conservative management of a single fetal death in twin pregnancy at a tertiary health institution in southern Nigeria. A case Report. IOSR Journal of Dental and Medical Sciences. 2014; 13 (3): 79-83.

[16] Cattanach SA, Wedel M, White S, Young M. Single Intrauterine Fetal Death in a suspected monozygotic Twin pregnancy. Aust N Z J Obstet Gynaecol. 1990; 30: 137-140.

[17] Prompeler HJ, Madjar H, Klosa W, du Bois A, Zahradnik HP, Schillinger $\mathrm{H}$, et al. Twin pregnancies with single fetal death. Acta Obstet Gynaecol Scand. 1994; 73: 205-208.

[18] Blickstein I, Perlman S. Single fetal death in twin gestations. J Perinat Med. 2013; 41: 65-69.

[19] Baweja KS, Pharmanand P. Continuation of pregnancy after abortion/delivery of first twin. J Obstet Gynaecol India. 2009; 59 (6): 573-575.

[20] Hillman SC, Morris RK, Kilby MD. Co-twin prognosis after single fetal death: a systematic review and meta-analysis. Obstet Gynaecol. 2011; 118: 928-940.

[21] Bajoria R, Wee LY, Anwar S, Ward S. Outcome of twin pregnancies complicated by single intrauterine death in relation to vascular anatomy of the monochorionic placenta. Hum Reprod. 1999; 14: 2124-2130.

[22] Landy HJ, Weingord AB. Management of multiple gestations complicated by an antepartum fetal demise. Obstet Gynecol Survey. 1989; 44: 171-176. 